\title{
Qlick Graphs with Crossing Number One
}

\author{
B. Basavanagoud* and V. R. Kulli ${ }^{\S}$ \\ *Department of Mathematics, Karnatak University, Dharwad - 580 003, Karnataka, India \\ ${ }^{\S}$ Department of Mathematics, Gulbarga University, Kalaburagi - 585 106, Karnataka, India \\ *email: b.basavanagoud@gmail.com, §email: vrkulli@gmail.com \\ *Corresponding Author.
}

Keywords: drawing, line graph, qlick graph, crossing number, forbidden subgraphs.

\begin{abstract}
In this paper, we deduce a necessary and sufficient condition for graphs whose qlick graphs have crossing number one. We also obtain a necessary and sufficient condition for qlick graphs to have crossing number one in terms of forbidden subgraphs.
\end{abstract}

\section{Introduction}

A graph is planar if it can be drawn in the plane or on the sphere in such a way that no two of its edges intersect. The crossing number $\operatorname{cr}(G)$ of a graph $G$ is the least number of intersections of pairs of edges in any embedding of $G$ in the plane. Obviously, $G$ is planar if and only if $\operatorname{cr}(G)=0$. It is implicit that the edges in a drawing are Jordan arcs (hence, non-selfintersecting), and it is easy to see that a drawing with the minimum number of crossing (an optimal drawing) must be a good drawing, that is, each two edges have at most one point in common, which is either a common end-vertex or a crossing.

All graphs considered here are finite undirected and without loops or multiple edges. We refer to [6] for unexplained terminology and notations. For a graph $G$, let $V(G), E(G)$ and $U(G)$ denote its vertex set, edge set and block set respectively. If $B=\left\{u_{1}, u_{2}, \ldots, u_{r} ; r \geq 2\right\}$ is a block of a graph $G$, then we say that the vertex $u_{1}$ and a block $B$ are incident with each other, as are $u_{2}$ and $B$ and so on. If $B=\left\{e_{1}, e_{2}, \ldots, e_{s} ; s \geq 1\right\}$ is a block of $G$, then we say that edge $e_{1}$ and block $B$ are incident with each other, as are $e_{2}$ and $B$ and so on. If two distinct blocks $B_{1}$ and $B_{2}$ are incident with common cut-vertex, then they are adjacent blocks. The edges and blocks of a graph are called its members.

The qlick graph $Q(G)$ of a graph $G$ is the graph whose set of vertices is the union of the set of edges $E(G)$ and set of blocks $U(G)$ of $G$ and in which two vertices are adjacent if and only if the corresponding edges are adjacent or corresponding blocks are adjacent or an edge and a block are incident. The plick graph $P(G)$ of $G$ is the graph whose vertex set is the set of edges $E(G)$ and set of blocks $U(G)$ of $G$ and two vertices are adjacent if and only if the corresponding edges are adjacent or an edge and a block are incident. These concepts were introduced by Kulli in [10] and were studied, for example, in $[1,2,11,12]$.

In [8], Kulli introduced the concept of total-block graph of a graph. In [3], Basavanagoud et al. generalized the concept of total-block graph and introduced the block-transformation graphs and defined as follows: Let $G=(V, E)$ be a graph with block set $U(G)$, and let $\alpha, \beta, \gamma$ be three variables having values 0 or 1 . The block-transformation graph $G^{\alpha \beta \gamma}$ is the graph having $V(G) \cup U(G)$ as the vertex set. For any two vertices $x$ and $y \in V(G) \cup U(G)$ we define $\alpha, \beta, \gamma$ as follows:

(i) Suppose $x, y$ are in $V(G) . \alpha=1$ if $x$ and $y$ are adjacent in $G . \alpha=0$ if $x$ and $y$ are nonadjacent in $G$. 
(ii) Suppose $x, y$ are in $U(G) . \beta=1$ if $x$ and $y$ are adjacent in $G$. $\beta=0$ if $x$ and $y$ are nonadjacent in $G$.

(iii) $x \in V(G)$ and $y \in U(G) . \gamma=1$ if $x$ and $y$ are incident with each other in $G$. $\gamma=0$ if $x$ and $y$ are nonincident with each other in $G$.

Thus, we obtain eight kinds of block -transformation graphs, in which $G^{111}$ is the total-block graph of $G$ [8], and $G^{000}$ is its complement. Also, $G^{001}, G^{010}$ and $G^{011}$ are the complements of $G^{110}$, $G^{101}$ and $G^{100}$ respectively. In [4], Basavanagoud et al. studied a criterian for (non-) planarity of the block-transformation graph $G^{\alpha \beta \gamma}$ when $\alpha \beta \gamma=101$.

The line graph $L(G)$ of a graph $G$ is the graph whose vertices can be put in one to one correspondence with the edges of $G$ in such a way that two vertices of $L(G)$ are adjacent if and only if the corresponding edges of $G$ are adjacent. The block graph $B(G)$ of a graph $G$ is the graph whose vertices are the blocks of $G$ and in which two vertices are adjacent whenever corresponding blocks have a cut-vertex in common. For other definitions concerning crossing numbers, line graphs or block graphs see [6].

The following will be useful in the proof of our results.

Remark 1.1 [11]. For any graph $G, L(G)$ and $B(G)$ are line disjoint subgraphs of $Q(G)$.

Theorem 1.1 [13]. The line graph of a planar graph $G$ is planar if and only if $\Delta(G) \leq 4$ and every vertex of degree 4 is a cut vertex.

Theorem 1.2 [7]. Let $G$ be a non planar graph. Then $\operatorname{cr}(L(G))=1$ if and only if the following conditions hold:

(1) $\operatorname{cr}(G)=1$;

(2) $\Delta(G) \leq 4$, and every vertex of degree 4 is a cut-vertex of $G$;

(3) There exists a drawing of $G$ in the plane with exactly one crossing in which each crossed edge is incident with a vertex of degree 2 .

Theorem 1.3 [9]. The line graph of a planar graph $G$ has crossing number one if and only if (1) or (2) holds:

(1) $\Delta(G)=4$ and there is unique non-cut-vertex of degree 4;

(2) $\Delta(G)=5$, every vertex of degree 4 is a cut-vertex, there is a unique vertex of degree 5 and it has at most 3 incident edges in any block.

Theorem 1.4 [11]. The qlick graph $Q(G)$ of a graph $G$ is planar if and only if $G$ satisfies the following conditions:

(1) $\Delta(G) \leq 3$, and

(2) if $\operatorname{deg}(v)=3$ for every vertex $v$ of $G$, then $v$ is a cut-vertex.

Theorem 1.5 [5] A graph has a planar line graph if and only if it has no subgraph homeomorphic to $K_{3,3}, K_{1,5}, P_{4}+K_{1}$ or $K_{2}+\overline{K_{3}}$.

\section{Results}

The following theorem supports the main theorem.

Theorem 2.1 Let $x$ be any edge of $K_{4}$. If $G$ is homeomorphic to $K_{4}-x$, then $\operatorname{cr}(Q(G))=1$.

Proof. We prove the theorem first for $K_{4}-x$. One can see that the graph $Q\left(K_{4}-x\right)$ has 6 vertices and 13 edges. But a planar graph with 6 vertices has at most 12 edges. This shows that $Q\left(K_{4}-x\right)$ has 
crossing number at least 1. Figure 1, being drawing of $Q\left(K_{4}-x\right)$ concludes that $\operatorname{cr}\left(Q\left(K_{4}-x\right)\right)=1$. Suppose now $G$ is the graph as in the statement. Referring to Figure 1, it is immediate to see that $\operatorname{cr}\left(Q\left(K_{4}-x\right)\right)=1$.

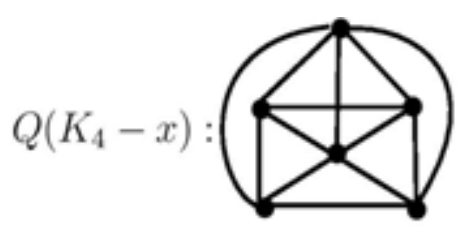

Figure 1

The following theorem gives a characterization of qlick graphs with crossing number 1 .

Theorem 2.2 A graph $G$ has a qlick graph with crossing number 1 if and only if $G$ is planar and one of the following holds:

(1) $\Delta(G)=3, G$ has exactly two adjacent non-cut-vertices of degree 3 and every other vertex of degree 3 is a cut-vertex.

(2) $\Delta(G)=4$, every vertex of degree 3 or 4 is a cut-vertex and there is a unique vertex $v$ of degree 4 and $v$ lies on 2 or 3 blocks.

Proof. Suppose $Q(G)$ has crossing number 1. Then by Remark 1.1, and by Theorem 1.2, $G$ is planar. By Theorem 1.4, $\Delta(G) \leq 4$, then there exists a non-cut-vertex of degree 3 or 4 and more than one vertex of degree 4 and $v$ lies on 2 or 3 blocks.

Suppose $\Delta(G) \leq 5$. Then $K_{1,5}$ is a subgraph of $G$. Clearly $L\left(K_{1,5}\right)=K_{5}$ and $B\left(K_{1,5}\right)=K_{5}$. It is known that $\operatorname{cr}\left(K_{5}\right)=1$. By remark 1.1, $2 K_{5}$ 's are line disjoint subgraphs of $Q(G)$ and hence $\operatorname{cr}(Q(G))>1$, a contradiction. This implies that $\Delta(G) \leq 4$. If $\Delta(G) \leq 2$, then by Theorem $1.4, Q(G)$ is planar, again a contradiction. Thus $\Delta(G)=3$ or 4 .

We now consider the following cases:

Case 1. Suppose $\Delta(G)=3$. Then by Theorem 1.4 and since $\operatorname{cr}(Q(G))=1, G$ has a non-cut-vertex of degree 3. Clearly $\mathrm{G}$ contains a subgraph homeomorphic to $K_{4}-x$, so that there exist at least two non-cut-vertex degree 3 . More precisely there is an even number, say $2 n$, of non-cut-vertex degree 3 . Now suppose $G$ has at least two diagonal edges. Then there are two subcases to consider depending on whether 2 diagonal edges exist in one cycle or in two different edge disjoint cycles.

Subcase 1.1. If two diagonal edges exist in one cycle of $G$. Then $G$ has a subgraph homeomorphic to $K_{4}$. The graph $Q\left(K_{4}\right)$ has 7 vertices and 18 edgers. It is known that a planar graph with 7 vertices has at most 15 edges. This shows that $Q\left(K_{4}\right)$ must have crossing number exceeding 1 and hence $Q(G)$ has crossing number greater than 1 , a contradiction.

Subcase 1.2. If two diagonal edges exist in two different edges-disjoint cycles of $G$. Then by Theorem 2.1, we see that for every subgraph of $G$ homeomorphic to $K_{4}-x$, there corresponds at least one crossing of $G$. Hence $Q(G)$ has at least 2 crossings, a contradiction. Hence $G$ has exactly two non-cut-vertices of degree 3 and every other vertex of degree 3 is a cut-vertex.

Suppose $G$ has two non-cut-vertices of degree 3 and they are not adjacent. Then $G$ contains a subgraph homeomorphic to $K_{2,3}$. On drawing $Q\left(K_{2,3}\right)$ in a plane one can see that $\operatorname{cr}\left(Q\left(K_{2,3}\right)\right)=2$. Since $Q\left(K_{2,3}\right)$ is a subgraph of $Q(G), Q(G)$ has crossing number exceeding 1, a contradiction.(see Figure 2) 
Therefore, we conclude that $G$ contains exactly two non-cut-vertices of degree 3 and these are adjacent. This proves(1).

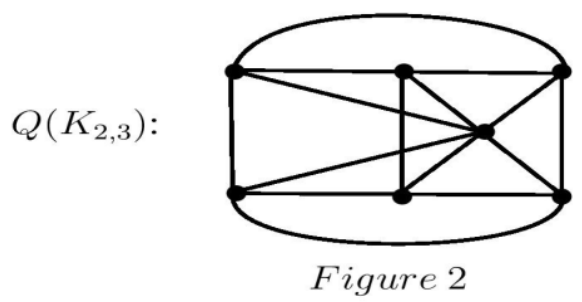

Case 2. Suppose $\Delta(G)=4$. Then every vertex of degree 3 is a cut-vertex, otherwise, by case $1, \mathrm{Q}(\mathrm{G})$ has crossing number exceeding 1 .

Next we show that every vertex of degree 4 is a cut-vertex. On the contrary suppose that $G$ has non-cut-vertex $v$ of degree 4 . Then by Theorem 1.3, $\operatorname{cr}(L(G)) \geq 1$. The vertex $u_{1}$ in $Q(G)$ corresponding to the block which contains a non-cut-vertex of degree 4 is adjacent to every vertex of $L(G)$. We obtain the drawing of $Q(G)$ with 3 crossings.

Assume $v$ lies on at least 4 blocks. The vertex $v$ and four of its neighboring vertices lie in a subgraph of $G$ isomorphic to $K_{1,4}$. On drawing $Q\left(K_{1,4}\right)$ in a plane, one can see that $\operatorname{cr}\left(Q\left(K_{1,4}\right)\right)>1$. Since $Q\left(K_{1,4}\right)$ is a subgraph of $Q(G), Q(G)$ has crossing number exceeding 1, a contradiction.

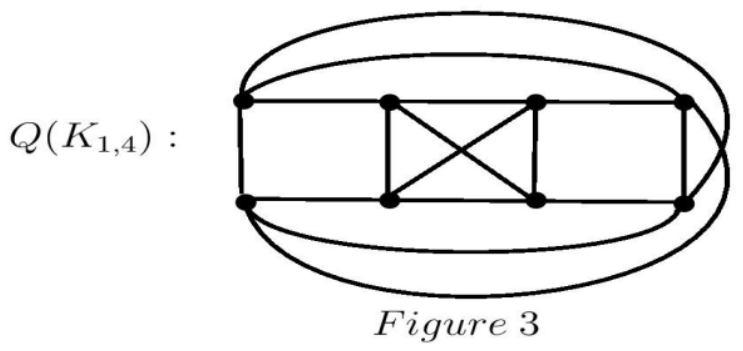

Suppose $G$ has at least two cut-vertices $u_{1}$ and $u_{2}$ which are of degree 4 . Then the cut-vertices $u_{1}$ and $u_{2}$ together with their corresponding incident four edges form two subgraphs as $K_{5}$ in $Q(G)$. Hence $\operatorname{cr}(Q(G))>1$, a contradiction.

Conversely, suppose $G$ is a planar graph satisfying (1) or (2). Then by Theorem 1.4, $Q(G)$ has crossing number at least 1 . We now show that its crossing number is at most 1 . First assume (1) holds. Then $G$ has exactly one subgraph $H_{1}$, homeomorphic to $K_{4}-x$ which contains exactly two adjacent non-cut-vertices of degree 3. By Theorem 2.1, $\operatorname{cr}\left(Q\left(H_{1}\right)\right)=1$. By Theorem 1.4, all other remaining blocks of $G$ have a planar qlick graphs. Hence $Q(G)$ has crossing number 1 .

Now assume (2) holds, and let $v$ be a unique cut-vertex of degree 4 and it lies on 2 or 3 blocks. We have the following cases.

Case 1. Suppose $v$ lies on 2 blocks. Then each block of $G$ is a cycle. On embedding of $Q(G)$ in a plane which gives $K_{3,3}$ as a subgraph in $Q(G)$ as shown in Fig 4 . Clearly $Q(G)$ has crossing number 1 . 


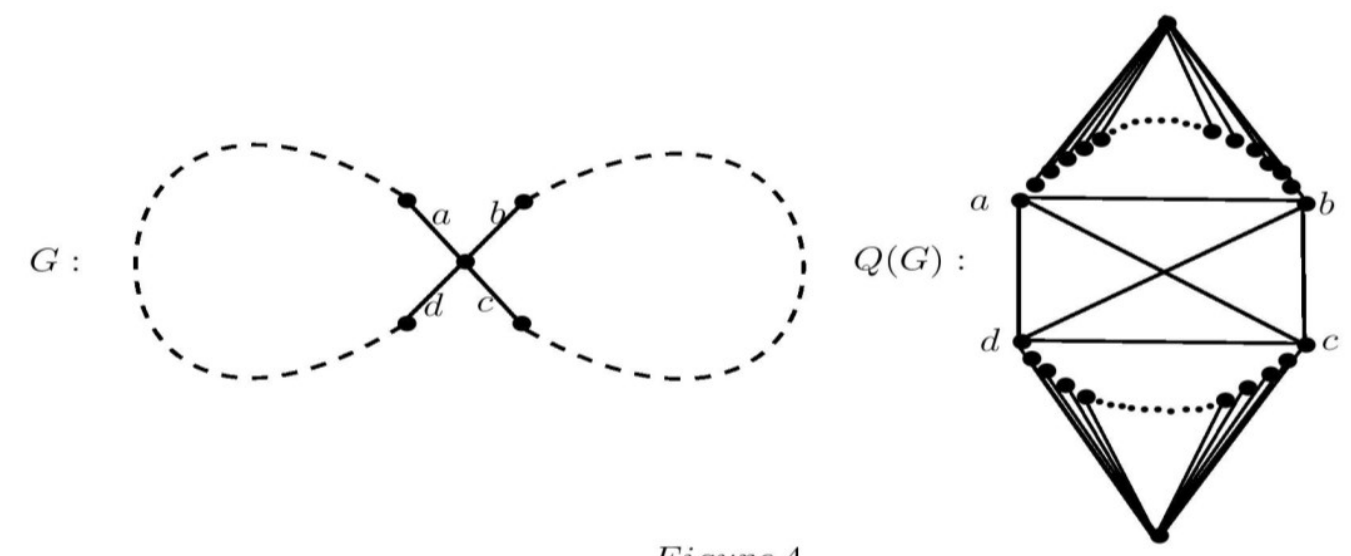

Figure 4.

Case 2. Suppose $v$ lies on 3 blocks. Then one of these blocks is a cycle and each of the remaining blocks is a $K_{2}$. The qlick graph $Q(G)$ can be constructed in the plane which gives exactly one subgraph $K_{5}$ (see Figure 5). Hence $\operatorname{cr}(Q(G))=1$.

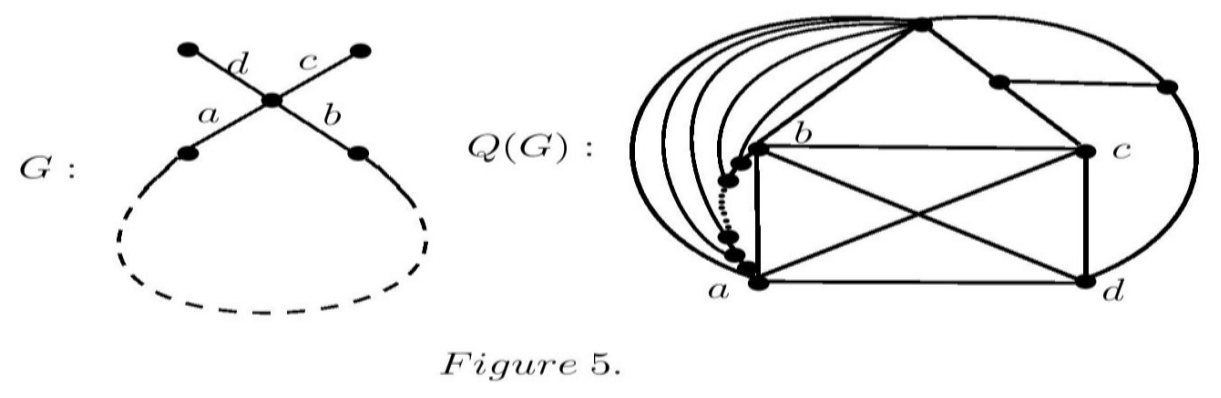

\section{Forbidden Subgraphs}

By using Theorem 2.2, we now characterize graphs whose qlick graphs have crossing number one in terms of forbidden subgraphs.

Theorem 3.1 The qlick graph $Q(G)$ of a connected graph $G$ has crossing number one if and only if $G$ has no induced subgraph $K_{1,4}$ or a subgraph homeomorphic from any one of the graphs Figure 6.
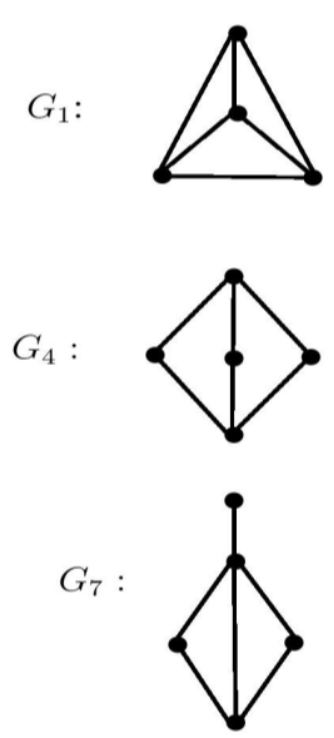

$G_{2}:$

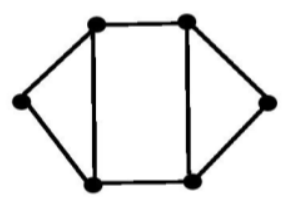

$G_{5}$ :
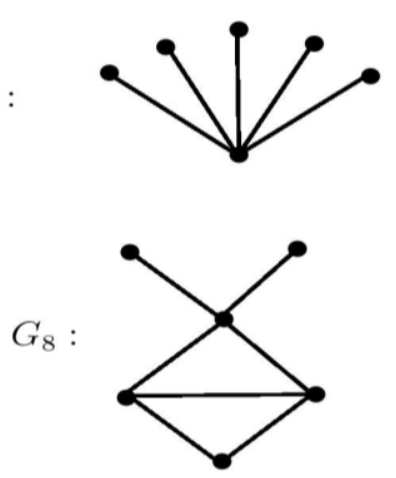

Figure 6

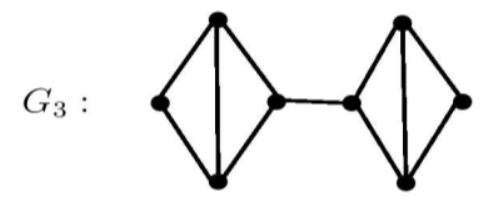

$G_{6}$ :

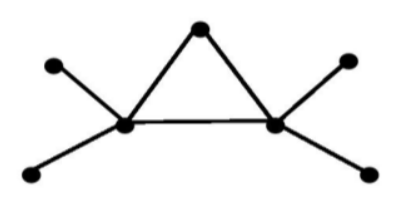

$G_{9}$ :

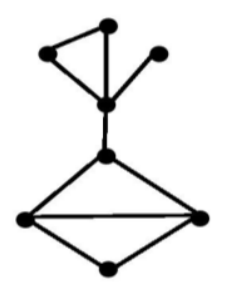


Proof. Suppose $G$ is a connected plane graph such that $Q(G)$ has crossing number one. By Theorem 2.2, we have (1) $\Delta(G)=3, G$ has exactly two adjacent non-cut-vertices of degree 3 and every other vertex of degree 3 is a cut-vertex or (2) $\Delta(G)=4$, every vertex of degree 3 or 4 is a cut-vertex and there is unique vertex $v$ of degree 4 lies on 2 or 3 blocks. From (1) and (2) it follows that $G$ has no induced subgraph $K_{1,4}$ or a subgraph homeomorphic from any one of the graphs of Fig 6 .

Conversely, suppose $G$ is a connected plane graph and it does not contain $K_{1,4}$ as induced subgraph or a subgraph homeomorphic from any one of the graphs of Fig 6. We now show that $G$ satisfies the condition (1) or (2) and hence by Theorem 2.1, $Q(G)$ has crossing number one. Suppose there exist at least three non-cut-vertices of degree 3 in $G$. Then $G$ has at least two diagonal edges. Here we consider two cases depending on whether 2 diagonal edges exist in one block or in two different blocks.

Case 1. If two diagonal edges exist in one block of $G$, then $G$ has a subgraph homeomorphic from $G_{1}$ or $G_{2}$.

Case 2. If two diagonal edges exist in two different blocks of $G$, then $G$ has a subgraph homeomorphic from $G_{3}$.

In each case we arrived at a contradiction. Hence $G$ has exactly two non-cut-vertices of degree 3 .

Suppose $G$ has exactly two nonadjacent non-cut-vertices of degree 3 . Then there exist 3 disjoint paths between these two non-cut-vertices of degree 3. Clearly $G$ contains a subgraph homeomorphic from $G_{4}$, a contradiction. Thus $G$ has exactly two adjacent non-cut-vertices of degree 3 .

Suppose $\Delta(G) \geq 5$. Then $G$ contains a subgraph homeomorphic from $G_{5}$, a contradiction. Hence $\Delta(G)=4$.

Let $v$ be a vertex of $G$ and $\operatorname{deg} v=4$. We prove that $v$ is a cut-vertex. On the contrary, let $a, b, c$ and $d$ be the vertices of $G$ adjacent to $v$. Then there exist paths between every pair of vertices of $a, b, c$ and $d$ not containing $v$. Then it is proved in Theorem 1.5, $G$ has a subgraph homeomorphic from $P_{4}+K_{1}$ or $K_{2}+\overline{K_{3}}$, since $P_{4}+K_{1}$ contains $G_{7}$ as a subgraph and $K_{2}+\overline{K_{3}}$ contains $G_{6}$ as a subgraph, this is a contradiction. Thus $v$ is a cut-vertex and every vertex of degree 4 is a cut-vertex.

Suppose every cut-vertex of degree 4 lies on 4 blocks of $G$. Let $v$ be the cut-vertex of degree 4 and it lies on 4 blocks. Then $K_{1,4}$ is an induced subgraph of $G$, a contradiction. Hence $v$ lies on 2 or 3 blocks of $G$.

Suppose there are at least two cut-vertices of degree 4 , each which lies 2 or 3 blocks. Let $v_{1}$ and $v_{2}$ be the two cut-vertices of degree 4 such that $v_{1}$ and $v_{2}$ are connected by a path $P$ and let $a_{i}$, $i=1,2,3$ and $b_{j}, j=1,2,3$ be the vertices of adjacent to $v_{1}$ and $v_{2}$ respectively.

We consider the following cases. 
Case 1. Assume $v_{1}$ and $v_{2}$ both lies on 3 blocks. If there exists a path between a vertex of $a_{i}$ and a vertex of $b_{j}$, then $G$ has a subgraph homeomorphic from $G_{6}$.

Case 2. Assume $v_{1}$ and $v_{2}$ both lies on 2 blocks. If there exists a path between a vertex of $a_{i}$ and a vertex of $b_{j}$ and there is a path between remaining two vertices of $a_{i}$ and two vertices of $b_{j}$, then $G$ contains $G_{6}$ as a subgraph.

We have exhausted all the cases and we arrive at the conclusion that $G$ has exactly one cut-vertex of degree 4 which lies on either 2 or 3 blocks of $G$.

Suppose deg $v=4$ and $v$ lies on 2 or 3 blocks of $G$ in which either of the blocks is neither a cycle nor a $K_{2}$. Then $G$ has a subgraph homeomorphic from $G_{7}$ or $G_{8}$.

Further suppose $\operatorname{deg} v_{1}=3$ for any other vertex $v_{1} \neq v$ of $G$ and $v_{1}$ is not a cut-vertex. The proof of the condition (2) of Theorem 2.2 will be completed if we disprove the above statement by deducing a contradiction.

We consider two cases.

Case 1. $v$ is adjacent to $v_{1}$.

Let $v_{2}$ and $v_{3}$ be the vertices of $G$ adjacent to $v_{1}$. Since $v_{1}$ is not a cut-vertex, there exist two shortest paths $Z_{1}\left(v-v_{3}\right)$ and $Z_{2}\left(v-v_{2}\right)$ which do not contain $v_{1}$. The minimality of the paths assures that either $v_{2} \notin Z_{1}$ or $v_{3} \notin Z_{2}$. Let $x$ be the first vertex of intersection (starting from $v_{2}$ ) of $Z_{2}$ with $Z_{1}$. If $x=v_{2}$ or $v_{3}$, then in either case $G$ has a subgraph homeomorphic from $G_{8}$ [see Fig.7( $\left.b_{1}\right)$ or $\left.7\left(b_{2}\right)\right]$. If $x=v$, then $G$ contains a subgraph homeomorphic from $G_{5}$ or $G_{7}$ [see Fig.7(c)]. If $x \neq v_{2}, v_{3}$ and $v$ then $G$ has a subgraph homeomorphic from $G_{8}$ [see Fig.7(a)].

Case 2. $v$ is not adjacent to $v_{1}$.

Since $G$ is connected, there exists a path $Z_{1}\left(v-v_{1}\right)$. Let $v_{2}$ and $v_{3}$ be the vertices of $G$ adjacent to $v_{1}$ and let $v_{4}$ be the vertex on $Z_{1}$ which divides $Z_{1}$ into subpaths $Z_{1}^{\prime}\left(v-v_{4}\right)$ and $Z_{1}^{\prime \prime}\left(v_{4}-v_{1}\right)$. Since $v_{1}$ is not a cut-vertex, there are two paths $Z_{2}\left(v_{4}-v_{2}\right)$ and $Z_{3}\left(v_{4}-v_{3}\right)$ which do not contain $v_{1}$. There are 4 subcases to consider depending on whether or not $v$ is on $Z_{2}$ and $Z_{3}$.

Subcase 2.1. $v$ is on $Z_{2}$ and $Z_{3}$.

Let $x$ be the last but one vertex of $Z_{2}$ which also belongs to $Z_{3}$. If $Z=v_{2}$ or $v_{3}$, then in either case we have a subgraph of $G$ that is homeomorphic from $G_{8}$ [see Fig.7( $\left.e_{1}\right)$ or 7.( $\left.e_{2}\right)$ ]. If $x=v$, then $G$ has a subgraph homeomorphic from $G_{5}, G_{7}$ or $G_{4}[$ see Fig.7 $(f)]$. If $x \neq v_{2}, v_{3}$ and $v_{4}$, then $G$ contains a subgraph homeomorphic from $G_{8}$ [see Fig.7(d)].

Subcase 2.2. $v$ lies on $Z_{2}$ or $Z_{3}$. In either case we have subgraph homeomorphic to $G_{8}$ [see Fig. $7\left(g_{1}\right)$ or $\left.7\left(g_{2}\right)\right]$.

Subcase 2.3. $v$ does not lies on $Z_{2}$ and $Z_{3}$. Again we consider two subcases of subcase 2.3. 
Subcase 2.3.1. $v$ lies on three blocks.

a] There exists a path (other than $\left.Z_{1}^{\prime}\right) v-y$ where $y \in Z_{1}^{\prime}$.

I] Assume $Z_{2}$ and $Z_{3}$ are not disjoint. Let $x$ be the last but one vertex of $Z_{2}$ which also belongs to $Z_{3}$. If $x=v_{2}$ or $v_{3}$, then in either case $G$ has a subgraph homeomorphic from $G_{8}$ or $G_{2}$ [see Fig. $7\left(i_{1}\right)$ or $\left.7\left(i_{2}\right)\right]$. If $x=v_{4}$, then $G$ contains a subgraph homeomorphic from $G_{8}, G_{7}$ or $G_{4}$ [see Fig.7(j) ]. If $x \neq v_{2}, v_{3}$ and $v_{4}$, then $G$ has a subgraph homeomorphic from $G_{8}, G_{2}$ or $G_{4}$ [see Fig.7(h) ]. If $y=v_{1}$ and $v_{1}, v_{3}$ are not adjacent, then $G$ has a subgraph homeomorphic from $G_{8}$ [see Fig.7(k)]. If $y \neq v_{1}$ and $v_{1}, v_{3}$ are not adjacent, then $G$ contains a subgraph homeomorphic from $G_{8}[$ see Fig. $7(l)]$.

$I I$ ] Assume $Z_{2}$ and $Z_{3}$ are disjoint. If $y=v_{1}$, then $G$ contains a subgraph homeomorphic from $G_{8}$ or $G_{4}$ [see Fig. $\left.7(m)\right]$. If $y \neq v_{1}$, then $G$ has a subgraph homeomorphic from $G_{7}, G_{8}$ or $G_{4}$ [see Fig. $7(n)]$.

b] There exists a path (other than $Z_{1}^{\prime \prime}$ ) $v-y$ where $y \in Z_{1}^{\prime \prime}$ or there exists a path between pair of adjacent vertices of $v$.

I] Assume $Z_{2}$ and $Z_{3}$ are not disjoint. Let $x$ be the last but one vertex of $Z_{2}$ which also belongs to $Z_{3}$. If $x=v_{2}$ or $v_{3}$, then in either case $G$ has a subgraph homeomorphic from $G_{9}$ [see Fig.7( $\left.p_{1}\right)$ or $7\left(p_{2}\right)$ ]. If $x \neq v_{2}, v_{3}$ and $v_{4}$, then $G$ has a subgraph homeomorphic from $G_{9}$ or $G_{4}$ [see Fig.7(o)].

$I I$ ] Assume $Z_{2}$ and $Z_{3}$ are adjacent. Then $x=v_{4}$. In this case $G$ has a subgraph homeomorphic from $G_{7}[$ see Fig. $7(q)]$.

We have completed all cases. In each case, we found that $G$ contains a subgraph homeomorphic from one of the forbidden subgraphs of Fig. 6 . Hence $v_{1}$ is a cut-vertex. Thus Theorem 2.2 implies that $G$ has a qlick graph with crossing number one. 


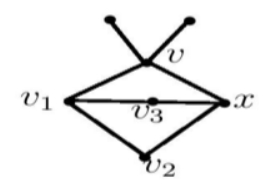

(a)

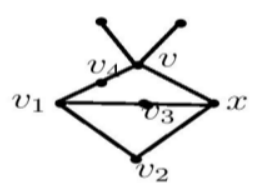

(d)

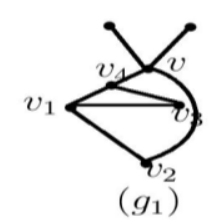<smiles>CC(C)C(C)C1CCCC1</smiles>

(i $\left.i_{2}\right)$

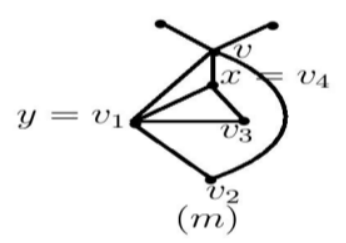

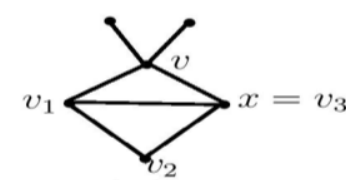

(b1)

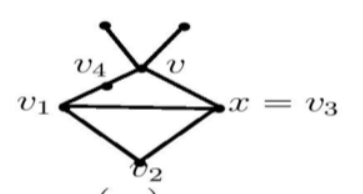

$\left(e_{1}\right)$
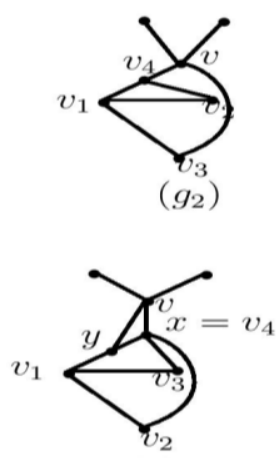

(j)
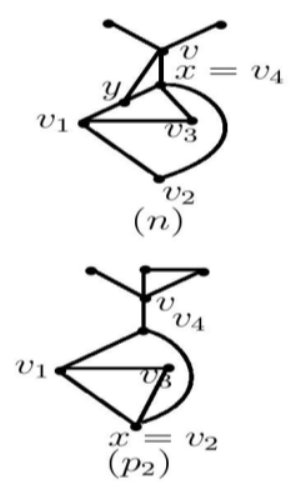
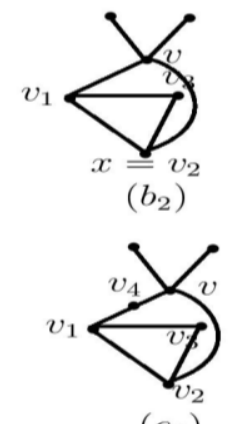

$\left(e_{2}\right)$

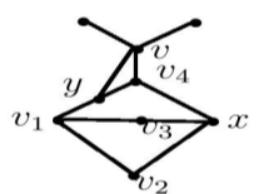

(h)

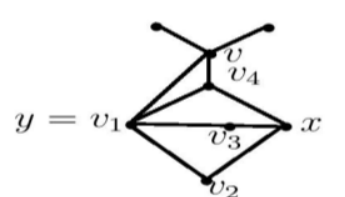

(k)

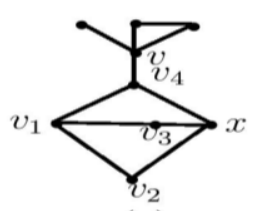

(o)

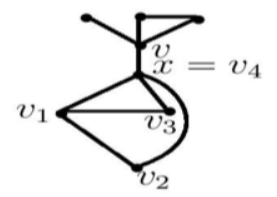

(q)<smiles>C[C@@H]1[C@H]2C=C[C@@H](C2)C1(C)C</smiles>

(c)<smiles>C[C@@H]1[C@H]2C=C[C@@H](C2)C1(C)C</smiles>

(f)

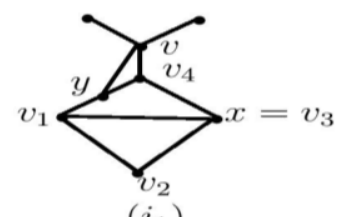

(i $)$

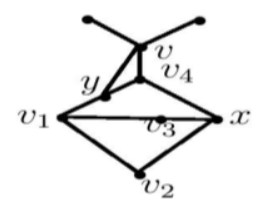

(l)

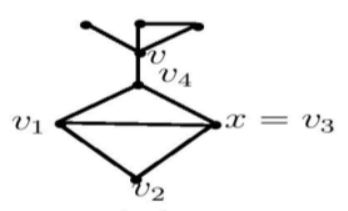

$\left(p_{1}\right)$

Figure 7

\section{Conclusion}

In this paper two characterizations are given for graphs whose qlick graphs have crossing number 1 , one in terms of degrees of vertices of a graph and the second in terms of forbidden subgraph. The crossing number $\operatorname{cr}(\mathrm{G})$ of $\mathrm{G}$ is the least number of intersections of pairs of edges in any embedding of $\mathrm{G}$ in the plane. Obviously $\mathrm{G}$ is planar if and only if $\operatorname{cr}(\mathrm{G})=0$.

\section{Acknowledgment}

The authors are thankful to the referee for his/her valuable suggestions. 


\section{References}

[1] B. Basavanagoud, V.R. Kulli, Hamiltonian and Eulerian properties of plick graphs, The Mathematics Student. 74 (2004) 175-181.

[2] B. Basavanagoud, V.R. Kulli, Plick graphs with crossing number 1, International J. Math. Combin. 2 (2011) 21-28.

[3] B. Basavanagoud, H.P. Patil, J.B. Veeragoudar, On the block-transformation graphs, graph equations and diameters, International Journal of Advances in Science and Technology. 2(2) (2011) 62-74.

[4] B. Basavanagoud, J.B. Veeragoudar, A criterion for (non-)planarity of the block-transformation graph $G^{\alpha \beta \gamma}$ when $\alpha \beta \gamma=101$, Bulletin of Mathematical Sciences and Applications, 10 (2014) $38-47$.

[5] D.L. Greenwell, R.L. Hemminger, Forbidden subgraphs for graphs with planar line graphs, Discrete Math. 2 (1972) 31-34.

[6] F. Harary, Graph Theory, Addison-Wesley, Reading, Mass, 1969.

[7] S. Jendrol, M. Klesc, On graphs whose line graphs have crossing number one, J. Graph Theory. 37 (2001) 181-188.

[8] V.R. Kulli, The semitotal-block graph and the total-block graph of a graph, Indian J. Pure Appl. Math. 7 (1976) 625-630.

[9] V.R. Kulli, D.G. Akka, L.W. Beineke, On line graphs with crossing number 1, J. Graph Theory. 3 (1979) 87-90.

[10] V.R. Kulli, On the plick graph and the qlick graph of a graph, Research Journal. 1 (1988) 48-52.

[11] V.R. Kulli, B. Basavanagoud, A criterion for (outer-) planarity of the qlick graph of a graph, Pure and Applied Mathematika Sciences. 48 (1998) 33-38.

[12] V.R. Kulli, B. Basavanagoud, Characterizations of planar plick graphs, Discussiones Mathematicae, Graph Theory. 24 (2004) 41-45.

[13] J. Sedlacek, Some propertices of interchange graphs, Theory of Graphs and its Applications, M. Fiedler, (Editor), Academic press, New York, 1964, pp. 145-150. 